\title{
A Taylor-Based Continuation Method for the Determination and Classification of Robot Singularities
}

\author{
Gauthier Hentz, Isabelle Charpentier, Lennart Rubbert \\ and Pierre Renaud
}

\begin{abstract}
Robot design in contexts such as computer-assisted medical interventions remains challenging. Compact, dexterous mechanisms with particular mobilities are needed, the synthesis of which requires a systematic evaluation of workspace and singular positions. The evaluation of singular positions and their classification are still difficult to perform in a systematic manner. In this paper, an automated method is presented and evaluated on a complex planar mechanism. A higher-order continuation method is used to provide continuous and accurate representation of singular locii. Classification is then performed by testing all the existing singularity types through a direct evaluation. Only the mechanism loop-closure equations are required thanks to automatic differentiation and the Diamanlab software developed for use of continuation. The evaluation of the method shows promising results.
\end{abstract}

\section{Introduction}

Robot design in contexts such as computer-assisted medical interventions remains challenging. Compact, dexterous mechanisms with particular mobilities are needed, which synthesis is today still an issue. In order to help the designer, efficient and automated methods are mandatory for mechanism assessment.

Workspace is a first property to be evaluated. We have described in [6] the use of automatic differentiation (AD) and the so-called Diamant higher-order continuation approach to compute the boundaries of reachable workspace in a simple manner. Built as an extension of [5], our approach is of interest for its high accuracy, low computation time and the description of workspace boundaries as Taylor series that yields a continuous representation.

The determination of mechanism singularities is another major requirement in mechanism evaluation. The problem is complex because of the variety of singular situations for a mechanism. Six singularity types exist and up to 21 different singularity classes can be detected during a mechanism analysis [10]. Several methods on

G. Hentz $(\varangle) \cdot$ I. Charpentier · L. Rubbert · P. Renaud

ICube, Illkirch-Graffenstaden, France

e-mail: gauthier.hentz@icube.unistra.fr 
singularity determination have been reported $[3,7,8]$. The determination is however usually not possible for all the singularity classes, and a general manipulator. In [10] algebraic and geometric techniques are given for finding singularity sets. Their application remains however tedious and time consuming. More recently, [1] proposed a general method based on interval analysis to perform an exhaustive numerical determination and classification of the singularities. The latter process relies on a sequential test process that can be affected by numerical accuracy issues, according to the authors.

In this paper, we investigate the extension of our continuation method [6] to propose a complete singularity analysis for general manipulators. Taking advantage of the continuity and accuracy properties of continuation, the method is based on a two-step process: firstly the determination of singularity locations, and secondly their classification. This latter phase is based on a direct evaluation of the 21 possible situations formulated in [10].

The paper is organized as follows. The singularity definition and classification is introduced in Sect. 2. The proposed determination and classification method is then detailed in Sect. 3. The method is tested in Sect. 4 by considering a planar mechanism with complex kinematic behavior. Conclusions on the potential of the method and future developments are detailed in Sect.5.

\section{Formulation of the Singularity Analysis Problem}

We consider here a mechanism requiring $N$ independent variables to define its configuration in a unique manner. Following [5], we choose to describe the mechanism configuration with a column-array $\mathbf{q}=\left[\mathbf{u}^{\top}, \mathbf{v}^{\top}, \mathbf{w}^{\top}\right]^{\top} \in \mathbb{R}^{n_{q}}$, with $\mathbf{u}, \mathbf{v}$ and $\mathbf{w}$ being respectively the output, input and passive coordinates. The mobility of the mechanism is denoted by $n$. We consider a non-redundant manipulator so that $\operatorname{dim}(\mathbf{u})=\operatorname{dim}(\mathbf{v})=n$ and $n_{q} \geq N+n$. The configuration space is the set of admissible values of $\mathbf{q}$ for the manipulator:

$$
\mathscr{C}=\left\{\mathbf{q} \mid \mathbf{R}^{\mathscr{C}}(\mathbf{q})=\mathbf{0}\right\},
$$

where $\mathbf{R}^{\mathscr{C}}(\mathbf{q})=\mathbf{0}$ is a system of $n_{e q}$ non-linear equations, typically the loop-closure equations for a parallel mechanism.

Feasible instantaneous motions are characterized by the velocity equations [10]:

$$
\mathbf{R}_{\mathbf{q}}^{\mathscr{C}}(\mathbf{q}) \dot{\mathbf{q}}=0
$$

with $\dot{\mathbf{q}}=\left[\dot{\mathbf{u}}^{\top}, \dot{\mathbf{v}}^{\top}, \dot{\mathbf{w}}^{\top}\right]^{\top}$ a column-array of velocities and $\mathbf{R}_{\mathbf{q}}^{\mathscr{C}}$ a $n_{e q} \times n_{q}$ Jacobian matrix. The following two kinematic problems are usually formulated: 
- Inverse instantaneous kinematics problem (IIKP): Find $\dot{\mathbf{q}}$ for a prescribed $\dot{\mathbf{u}}$.

- Forward instantaneous kinematics problem (FIKP): Find $\dot{\mathbf{q}}$ for a prescribed $\dot{\mathbf{v}}$.

Singularities can be defined as indeterminations in these two problems. To express that, let sets of variables $\mathbf{z}=\left[\mathbf{v}^{\top}, \mathbf{w}^{\top}\right]^{\top}$ and $\mathbf{y}=\left[\mathbf{u}^{\top}, \mathbf{w}^{\top}\right]^{\top}$ be two sets of variables issued from $\mathbf{q}$. We note $\mathbf{R}_{\mathbf{z}}^{\mathscr{C}}, \mathbf{R}_{\mathbf{y}}^{\mathscr{C}}$ and $\mathbf{R}_{\mathbf{w}}^{\mathscr{C}}$ the submatrices obtained from $\mathbf{R}_{\mathbf{q}}^{\mathscr{C}}$ by removal of the columns related to $\mathbf{u}, \mathbf{v}$ and $(\mathbf{u}, \mathbf{v})$, respectively [1].

Indetermination situations of the IIKP occur when $\mathbf{R}_{\mathbf{z}}^{\mathscr{C}}$ is rank deficient. The configurations $\mathbf{q} \in \mathscr{S}_{\mathbf{z}} \subset \mathscr{C}$ where these situations occur are called inverse singularities and are solutions [5] of:

$$
\left[\begin{array}{c}
\mathbf{R}^{\mathscr{C}}(\mathbf{u}, \mathbf{z}) \\
\left(\mathbf{R}_{\mathbf{z}}^{\mathscr{C}}(\mathbf{u}, \mathbf{z})\right)^{\top} \\
\xi^{\top} \xi-1
\end{array}\right]=0
$$

where $\xi \in \mathbb{R ^ { n _ { e q } }}$. The two lower terms express the rank deficiency of the Jacobian $\mathbf{R}_{\mathbf{z}}^{\mathscr{C}}$. Indetermination situations of the FIKP constitute a set $\mathscr{S}_{\mathbf{y}}$ of solutions to

$$
\left[\begin{array}{c}
\mathbf{R}^{\mathscr{C}}(\mathbf{v}, \mathbf{y}) \\
\left(\mathbf{R}_{\mathbf{y}}^{\mathscr{C}}(\mathbf{v}, \mathbf{y})\right)^{\top} \xi \\
\xi^{\top} \xi-1
\end{array}\right]=0 .
$$

Solutions to systems (3) and (4) define all singularities of a mechanism [1].

In order to express the nature of singularities for a mechanism, a classification has to be performed. A classification of the singularities in 6 singularity types was introduced in [10] with redundant input (RI), redundant output (RO), redundant passive motion (RPM), impossible input (II), impossible output (IO), and increased instantaneous motion (IIM) singularities. A set of 6 criteria allows such classification [10]:

$$
\begin{array}{ll}
\text { - } \mathbf{q} \in\{R I\} \Leftrightarrow \operatorname{rank}\left(\mathbf{R}_{\mathbf{z}}^{\mathscr{C}}\right)<\operatorname{rank}\left(\mathbf{R}_{\mathbf{w}}^{\mathscr{C}}\right)+n & \bullet \mathbf{q} \in\{I I\} \Leftrightarrow \operatorname{rank}\left(\mathbf{R}_{\mathbf{y}}^{\mathscr{C}}\right)<\operatorname{rank}\left(\mathbf{R}_{\mathbf{q}}^{\mathscr{C}}\right) \\
\text { - } \mathbf{q} \in\{R O\} \Leftrightarrow \operatorname{rank}\left(\mathbf{R}_{\mathbf{y}}^{\mathscr{C}}\right)<\operatorname{rank}\left(\mathbf{R}_{\mathbf{w}}^{\mathscr{C}}\right)+n & \bullet \mathbf{q} \in\{I O\} \Leftrightarrow \operatorname{rank}\left(\mathbf{R}_{\mathbf{z}}^{\mathscr{C}}\right)<\operatorname{rank}\left(\mathbf{R}_{\mathbf{q}}^{\mathscr{C}}\right) \\
\text { - } \mathbf{q} \in\{R P M\} \Leftrightarrow \operatorname{rank}\left(\mathbf{R}_{\mathbf{w}}^{\mathscr{C}}\right)<N-n & \bullet \mathbf{q} \in\{I I M\} \Leftrightarrow \operatorname{rank}\left(\mathbf{R}_{\mathbf{q}}^{\mathscr{C}}\right)<N
\end{array}
$$

A singular position can be of several types at the same time. A total of 21 classes exists [10], each one corresponding to a particular degenerate kinematic behavior of the mechanism. Indetermination of either the IIKP or the FIKP are the most common and studied cases and correspond respectively to the well known output (also designated as serial or (RI,IO)) singularities or input (also designated as parallel or (RO,II)) singularities. Configurations satisfying both systems (3) and (4) can belong to any of the other singularity classes. The corresponding singularity sets are typically of lower dimension than the whole singularity set, implying different kinematic behavior [1]. 


\section{A Fully Automated Computation Method}

The method we propose is based on computation of singularities followed by their classification.

\section{Determination of the Singularity Set}

The solutions to the systems (3) and (4) are composed of several continuous sets, that can be designated as solution branches. We propose to follow solution branches from an initial point by a numerical continuation technique. Intersections between solution branches can occur. This corresponds to so-called bifurcation points. At such points, the continuity of the solution set is broken and rank deficiency of the Jacobian of the system occurs [9]. The detection of such points allows us to locate branch intersections. A branch switching may then be carried out, allowing a complete determination of all the connected branches from one initial point. If the singularity set is one-dimensional, continuation can be directly applied. If not, linear relationships between the coordinates can be added to discretize the determination of solution sets, as described in [5] and used in [6].

With a classical first-order continuation technique, branches are computed stepby-step as a collection of solution points. With higher-order continuation, the branches of solutions are computed as Taylor series which constitute continuous and accurate representations. The system to be solved needs however a formulation that usually requires complex and time-consuming additional work. On the contrary, Diamant $^{1}$ [2], the numerical method we use, is an implementation of higher-order continuation with integration of automatic differentiation (AD). Using this framework, non-linear equations describing a mechanism behavior are directly implemented in their standard format and are being solved in a fully automated manner. Bifurcation detection and branch switching are carried out automatically as well. In addition, the Tapenade software [4] is used to build the Jacobian $\mathbf{R}_{\mathbf{q}}^{\mathscr{C}}$ from system (1) at no cost, benefiting again from AD. Finally our method builds on AD and Diamant to propose a fully automated framework for singularity analysis, allowing to generate and solve systems (3) or (4) in a fully automated manner. The reader is referred to [6] and the references therein for further explanations on AD and Diamant.

In addition to this exploration technique of the singularity sets, we propose to monitor the rank deficiency of $\mathbf{R}_{\mathbf{y}}^{\mathscr{C}}$ (respectively $\mathbf{R}_{\mathbf{z}}^{\mathscr{C}}$ ) during the determination of solutions of (3) (respectively (4)). In this way, if a rank deficiency of the monitored Jacobian occurs, the corresponding configuration is located at the intersection of $\mathscr{S}_{\mathbf{z}}$ and $\mathscr{S}_{\mathbf{y}}$. We can therefore identify the input and output singularities and also the other types of singularities. This means we can identify precisely and immediately configurations that can belong to any of the 21 singularity classes. When the described

\footnotetext{
${ }^{1}$ Stands for Différentiation Automatique de la Méthode Asymptotique Numérique Typée.
} 
rank deficiency is detected, we can in addition use the corresponding points to switch from branches of $\mathscr{S}_{\mathbf{z}}$ to branches of $\mathscr{S}_{\mathbf{y}}$ and conversely. There is then no need for another initial point to compute all the connected branches of $\mathscr{S}_{\mathbf{z}} \cup \mathscr{S}_{\mathbf{y}}$.

Thanks to AD, Diamant, monitoring of rank deficiency and automated branch switching, we obtain the whole connected singularity set $\mathscr{S}_{\mathbf{z}} \cup \mathscr{S}_{\mathbf{y}}$ as a grid of solution branches connected by singular points. The determination step can be finally described as:

1. Determination:

a. Automatic differentiation of system (1) with respect to (u, v, w) using Tapenade software [4] to build the Jacobian $\mathbf{R}_{\mathbf{q}}^{\mathscr{C}}$ and the submatrices $\mathbf{R}_{\mathbf{z}}^{\mathscr{C}}, \mathbf{R}_{\mathbf{y}}^{\mathscr{C}}$ and $\mathbf{R}_{\mathbf{w}}^{\mathscr{C}}$

b. Computation of the singularities by solving systems (3) and (4) with the non-linear solver Diamant [2]. Taylor series allow for the local construction of solution branches. Monitoring of the rank deficiency of $\mathbf{R}_{\mathbf{y}}$ during continuation on system (3), and conversely, allows to detect points of $\mathscr{S}_{\mathbf{z}} \cap \mathscr{S}_{\mathbf{y}}$.

\section{Singularity Classification}

The solution branches of either (3) or (4) can be directly classified respectively in (RI,IO) or (RO,II) singularity classes. Other detected singular points can belong to any of the other existing singularity classes, which requires additional classification steps. Thanks to the high accuracy in the estimation of singular points, a direct classification of the solutions of $\mathscr{S}_{\mathbf{z}} \cap \mathscr{S}_{\mathbf{y}}$ can be performed. Each point is tested from the propositions given in Sect. 2 . The classification step can be finally described as:

2. Classification of the points detected in $\mathscr{S}_{\mathbf{z}} \cap \mathscr{S}_{\mathbf{y}}$ using the test functions. For each point:

a. Computation of the ranks of matrices $\mathbf{R}_{\mathbf{q}}^{\mathscr{C}}, \mathbf{R}_{\mathbf{w}}^{\mathscr{C}}, \mathbf{R}_{\mathbf{y}}^{\mathscr{C}}$ and $\mathbf{R}_{\mathbf{z}}^{\mathscr{C}}$,

b. Determination of the singularity types from the criteria given in Sect. 2,

c. Determination of the singularity class (see Table 1 in [10]) by testing in a successive manner membership to (a) combinations of RI, RO, RPM types (rows of the Table), (b) IIM type, (c) IO type and (d) II type. 


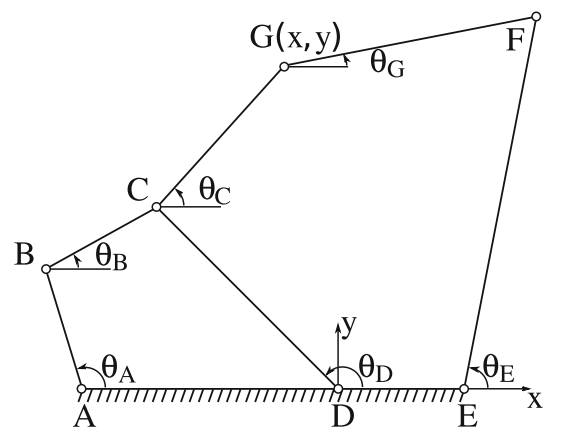

(a) Kinematic scheme

\begin{tabular}{|c|c|}
\hline \multicolumn{1}{|c|}{ Singularity classes } \\
\hline & $(\mathrm{RI}, \mathrm{IO})$ \\
& $(\mathrm{RO}, \mathrm{II})$ \\
$\times$ & $(\mathrm{RI}, \mathrm{RO}, \mathrm{IIM})$ \\
\hline & $(\mathrm{RI}, \mathrm{IO}),(\mathrm{RO}, \mathrm{II})$ \\
& $(\mathrm{RI}, \mathrm{RO}, \mathrm{II}, \mathrm{IO})$ \\
+ & $(\mathrm{RI}, \mathrm{RPM}, \mathrm{IO}, \mathrm{IIM})$ \\
\hline
\end{tabular}

(b) Singularity classes

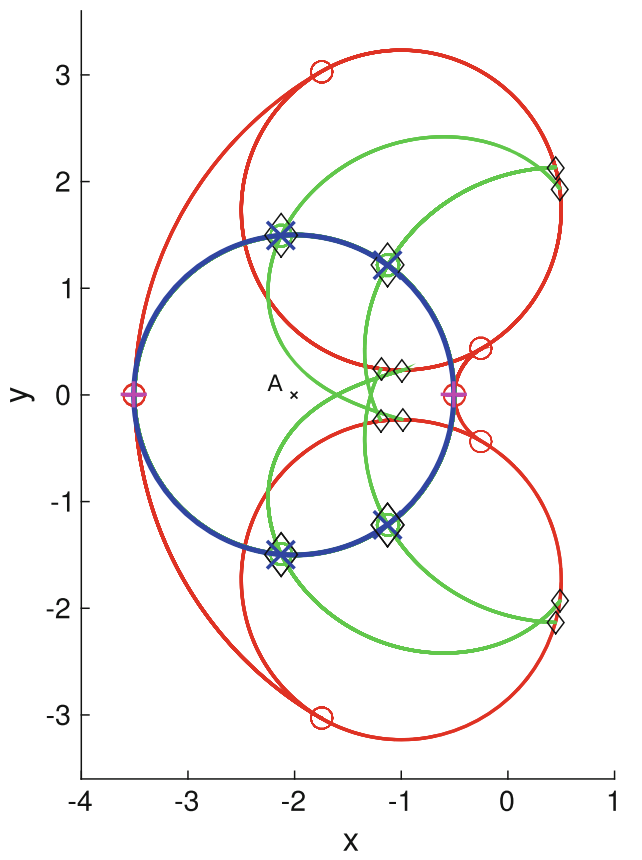

(c) Set $\mathscr{S}_{\mathbf{z}} \cup \mathscr{S}_{\mathbf{y}}$ obtained with our method.

Fig. 1 Singularity set (c) of the double-loop mechanism depicted in (a) after projection onto the space of the $x$ and $y$ variables. Each singularity in the configuration space $\mathscr{C}$ is classified according to the singularity classes indicated in (b)

\section{Application to a 2-DOF Double-Loop Manipulator}

\section{Manipulator Description}

In order to assess the efficiency of the method, the 2-DOF planar manipulator depicted in Fig. 1a is considered. This mechanism is a double-loop parallel mechanism, and it possesses two mobilities $(n=2)$. In terms of singularity analysis, it is very challenging, especially with the geometric parameters issued from [1], for which all existing singularity types occur. Its planar nature allows at the same time a comprehensive interpretation of the kinematic behavior implied by each class of singularity, see [1], which makes it a very interesting case study for the method benchmark.

The constraint equations describing the mechanism kinematics, adapted from [1], are obtained by writing the closure equations for both loops and two equations that constrain the position of point $G$ :

$$
\mathbf{R}^{\mathscr{C}}(\mathbf{q})=\left[\begin{array}{l}
C D \cos \left(\theta_{D}\right)+C G \cos \left(\theta_{C}\right)-x \\
C D \sin \left(\theta_{D}\right)+C G \sin \left(\theta_{C}\right)-y \\
A B \cos \left(\theta_{A}\right)+B C \cos \left(\theta_{B}\right)-C D \cos \left(\theta_{D}\right)-A D \\
A B \sin \left(\theta_{A}\right)+B C \sin \left(\theta_{B}\right)-C D \sin \left(\theta_{D}\right) \\
C D \cos \left(\theta_{D}\right)+C G \cos \left(\theta_{C}\right)+F G \cos \left(\theta_{G}\right)-E F \cos \left(\theta_{E}\right)-D E \\
C D \sin \left(\theta_{D}\right)+C G \sin \left(\theta_{C}\right)+F G \sin \left(\theta_{G}\right)-E F \sin \left(\theta_{E}\right)
\end{array}\right]=\mathbf{0}
$$


with $\theta_{A}$ and $\theta_{E}$ the actuated joint coordinates, $x$ and $y$ the coordinates of point $G$. The selected passive coordinates are $\theta_{B}, \theta_{C}, \theta_{D}$ and $\theta_{G}$. Geometric parameters are defined as $A B=B C=D E=1, A D=C D=F G=2, C G=1.5, E F=3$.

\section{Results and Interpretation}

The implementation is performed using Taylor series at a truncated order of 20 and a precision of $1 e-6$ on the residual [2]. Eigenvalues of square matrices and singular values of rectangular matrices are considered null if inferior to a threshold of $1 e-5$. The solution branches corresponding to the output and input singularity locii are defined in the configuration space $\mathscr{C}$. For sake of representation they are projected onto the space of the $x$ and $y$ variables only and plotted in Fig. 1c, using respectively green and red lines.

Bifurcation points detected automatically during the continuation process are indicated with a circle-shape marker (o). They allow us to detect branch intersections in $\mathscr{S}_{\mathbf{z}}$ or $\mathscr{S}_{\mathbf{y}}$. Intersection points between the output and input singularity sets, detected by monitoring the simultaneous rank deficiency of both $\mathbf{R}_{\mathbf{z}}$ and $\mathbf{R}_{\mathbf{y}}$, are indicated by the other markers. As expected, detection of the intersection points and of the bifurcation points allow for the computation of all connected solution branches of $\mathscr{S}_{\mathbf{z}} \cup \mathscr{S}_{\mathbf{y}}$. All the singularities other than input or output singularities are then classified by using the second step of the method.

The accuracy in the determination of the branches and the location method allows us to assign a detected point to the class it belongs to in a straightforward manner. All connected singularities can thus be entirely determined and classified. Markers using multiplication symbol $(\times)$ designate $(\mathrm{RI}, \mathrm{RO}, \mathrm{IIM})$ configurations. The points depicted as diamonds $(\diamond)$ correspond to simultaneous output and input singularities (RI,RO,II,IO). Two points belonging to (RI,RPM,IO,IIM) class are indicated with plus-shape markers $(+)$. Some points on the projected diagram are referenced with several classes. This means they correspond to several configurations in $\mathscr{C}$, because a configuration must belong to a unique class [10]. The blue circle of center $A$ is in particular the superimposition of (RI,IO) and (RO,II) branches. The known singularities are obtained, at the exception of singularities that are superimposed to the blue circle in Fig. 1c (see also [1], Fig.4). Further analysis shows that the gain of mobility in that situation would require an exploration with continuation along an additional direction. This would require as described earlier the introduction of a discretization and an additional relationship in the system, a development to be investigated.

\section{Discussion and Conclusion}

In this paper, we have introduced the use of higher-order continuation for accurate and automatic determination of singularity sets. The accuracy of Taylor series computation together with automatic differentiation allows us to easily implement the singularity determination problem from loop-closure equations and perform an 
unambiguous classification of the obtained singularities. This is of particular interest for the designer since only very little information on the mechanism is needed to conduct the analysis.

The evaluation is promising, with the estimation of all input and output singularities, and more complex situations as described in Fig. 1. Only one set of IIM singular positions is today not determined. Their estimation requires an improvement of the exploration strategy by continuation that will be investigated.

Future work will be focused on the evaluation of the method by considering other 3D mechanisms. It will be also interesting to combine our method with the intervalbased method from [1] to get accurate and efficient determination of singularities as well as an exhaustive determination, even in difficult cases of disconnected sets, without any initial knowledge on the singular configurations. Sensitivity analysis also provided by the Diamant framework will then also be exploited to provide the designer with a tool for optimization of the mechanism geometry.

Acknowledgements This work was supported by the French government research program Investissements d'avenir through the Robotex Equipment of Excellence (ANR-10-EQPX-44).

\section{References}

1. Bohigas, O., Zlatanov, D., Manubens, M., Ros, L.: On the numerical classification of the singularities of robot manipulators. In: ASME 2012 International Design Engineering Technical Conferences, pp. 1287-1296 (2012)

2. Charpentier, I.: On higher-order differentiation in nonlinear mechanics. Optim. Methods Softw. 27, 221-232 (2012)

3. Gosselin, C., Angeles, J.: Singularity analysis of closed-loop kinematic chains. IEEE Trans. Robot. Autom. 6(3), 281-290 (1990)

4. Hascoet, L., Pascual, V.: The Tapenade automatic differentiation tool: principles, model, and specification. ACM Trans. Math. Softw. 39, 20:1-20:43 (2013)

5. Haug, E.J., Luh, C.M., Adkins, F.A., Wang, J.Y.: Numerical algorithms for mapping boundaries of manipulator workspaces. ASME J. Mech. Design 118, 228-234 (1996)

6. Hentz, G., Charpentier, I., Renaud, P.: Higher-order continuation for the determination of robot workspace boundaries. Comptes Rendus Mécanique 344(2), 95-101 (2016)

7. Merlet, J.P.: A formal-numerical approach for robust in-workspace singularity detection. IEEE Trans. Robot. 23(3), 393-402 (2007)

8. Park, F., Kim, J.W.: Singularity analysis of closed kinematic chains. J. Mech. Design 121(1), 32-38 (1999)

9. Seydel, R.: Practical Bifurcation and Stability Analysis, 3rd edn. Springer, Berlin (2009)

10. Zlatanov, D., Fenton, R.G., Benhabib, B.: A unifying framework for classification and interpretation of mechanism singularities. ASME J. Mech. Design 117(4), 566-572 (1995) 